\title{
Microfluidics as a tool for C. elegans research
}

\author{
Adriana San-Miguel and Hang $\mathrm{Lu}^{\mathcal{S}}$ \\ School of Chemical \& Biomolecular Engineering, Georgia Institute of Technology, Atlanta GA, \\ 30332, USA
}

\section{Table of Contents}

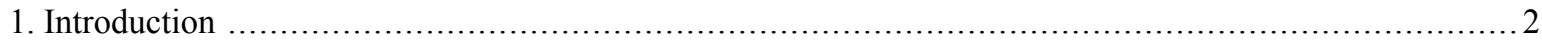

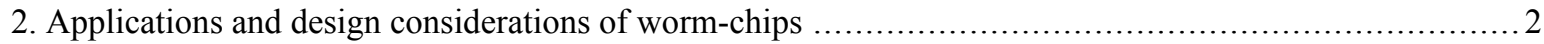

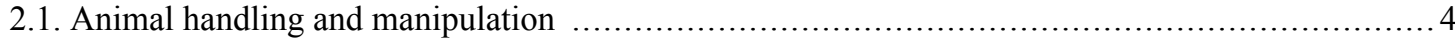

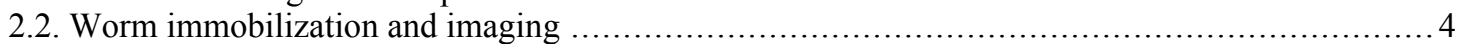

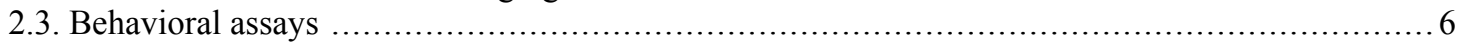

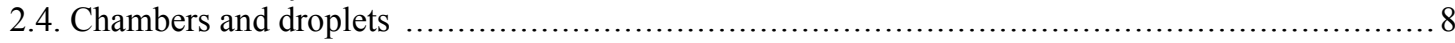

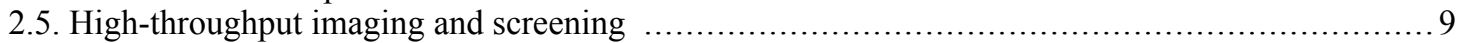

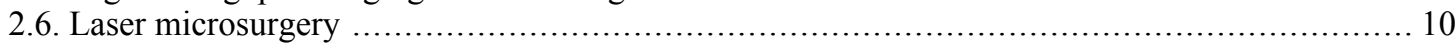

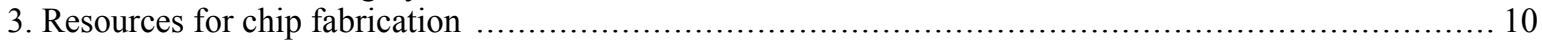

4. Fabrication of microfluidic devices for C. elegans studies with soft lithography in-house. ............... 10

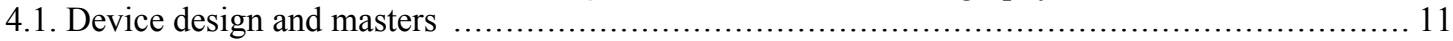

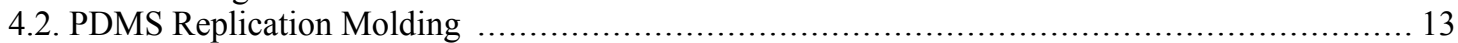

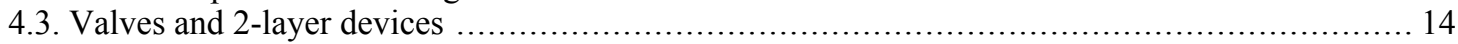

4.4. Bonding the devices and connections to the macro world ...................................... 15

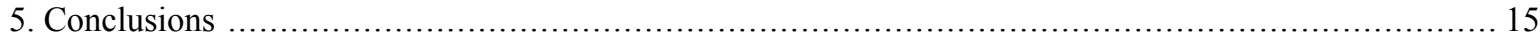

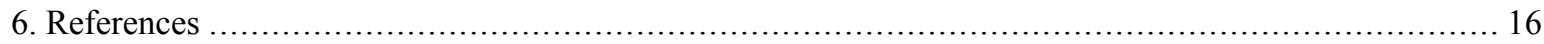

Abstract:

Microfluidics has emerged as a set of powerful tools that have greatly advanced some areas of biological research, including research using $C$. elegans. The use of microfluidics has enabled many experiments that are otherwise impossible with conventional methods. Today there are many examples that demonstrate the main advantages of using microfluidics for $C$. elegans research, achieving precise environmental conditions and facilitating worm handling. Examples range from behavioral analysis under precise chemical or odor

\footnotetext{
${ }^{*}$ Edited by Oliver Hobert Last revised: January 2, 2013, Published September 24, 2013. This chapter should be cited as: San-Miguel A. and Lu H. Microfluidics as a tool for C. elegans research. (September 24, 2013), WormBook, ed. The C. elegans Research Community, WormBook, doi/10.1895/wormbook.1.162.1, http://www.wormbook.org.

Copyright: (C) 2013 Adriana San-Miguel and Hang Lu. This is an open-access article distributed under the terms of the Creative Commons Attribution License, which permits unrestricted use, distribution, and reproduction in any medium, provided the original author and source are credited.

${ }^{\S}$ To whom correspondence should be addressed. E-mail: hang.lu@gatech.edu
} 
stimulation, locomotion studies in well-defined structural surroundings, and even long-term culture on chip. Moreover, microfluidics has enabled coupling worm handling and imaging thus facilitating genetic screens, optogenetic studies, and laser ablation experiments. In this article, we review some of the applications of microfluidics for $C$. elegans research and provide guides for the design, fabrication, and use of microfluidic devices for $C$. elegans research studies.

\section{Introduction}

Microfluidics has recently emerged as a new technology for studies at the small scale ranging from the biological sciences, soft matter, reactions and synthesis, high-throughput drug screening, encapsulation, and lab-on-chip diagnostics (Whitesides, 2006). As the name suggests, microfluidics involves fluid flows at the micron-scale, which are easily predictable and controllable due to low Reynolds numbers (Bretscher et al., 2008). Consequently, accurate flow rates, concentration gradients, and shear rates can be applied in experiments, which additionally require very small sample sizes or reagent volumes. Moreover, the large surface-to-volume area characteristic of micron-scale features allows fast mass and heat transfer.

Microfluidic devices evolved from the microfabrication methods used for electronic chips and MicroElectroMechanical Systems (MEMS), which produce devices with small features ranging from 1 to $100 \mu \mathrm{m}$ with high precision. Typically, simple microfluidic devices are made by soft lithography replica molding of a flexible silicone elastomer, PDMS (polydimethylsiloxane) (Duffy et al., 1998; McDonald et al., 2000; Xia and Whitesides, 1998). This material is optically transparent, gas permeable, non-toxic, inert, and compatible with aqueous solutions, making it suitable for biological experimentation and microscopic observation. Fabrication of microfluidic devices is easy, fast and inexpensive, and devices are thus usually single-use.

Because of all the advantages it offers, the use of microfluidics as a platform for biological studies has been greatly increased. For instance, microfluidics has been applied to study cells (Andersson and van den Berg, 2003; Chung et al., 2011b) as well as whole organisms such as Drosophila melanogaster (Chung et al., 2011a; Lucchetta et al., 2005). C. elegans research has been particularly impacted by this technology (Albrecht and Bargmann, 2011; Ben-Yakar et al., 2009; Chalasani et al., 2007; Chronis, 2010; Chronis et al., 2007; Crane et al., 2010; Gray et al., 2004; Hulme et al., 2008; Lockery, 2007; Lockery et al., 2008; McCormick et al., 2011; Yanik et al., 2011; Zhang et al., 2005; Zimmer et al., 2009), as it not only allows controlled flows and chemical stimuli delivery, but it also provides a platform for easy animal handling and manipulation, which is otherwise manual, laborious, and time-consuming. Additionally, the use of glues and anesthetics for studies that require worm immobilization can be avoided. Some devices have been used to examine behavior and neuronal activity under certain spatial or temporal stimulus. The study of sensory and locomotion behavior in mazes and patterned surfaces has also been made possible by microfluidics (Lockery et al., 2008; Zhang et al., 2005). Moreover, high-throughput imaging, phenotyping and screening, as well as laser ablation and nerve regeneration studies have been successfully performed with the help of this technology (Chung and Lu, 2009; Chung et al., 2008; Crane et al., 2009; Crane et al., 2010; Guo et al., 2008; Rohde et al., 2007; Stirman et al., 2010). In general, engineering research in microfluidics has aimed at potentially transforming $C$. elegans research from a laborious time-consuming per-worm experimenting platform into an easier task, aided by the simple, precise and rapid on-chip worm handling, immobilization, and imaging.

Microfluidic devices for worm studies can be made in a single layer or in multiple layers, with valves or without them, with square or round channels, and with structures as simple as a channel or with very complicated features of only a few microns in size. Additionally they can incorporate elements for efficient immobilization and worm trapping. Furthermore, devices with multiple-depth features can also be made. This article describes the main applications of worm-chips developed thus far as well as the basics of fabrication and functioning of simple one-layer PDMS devices for C. elegans research, which can be easily fabricated by non-expert users. An optimal design for each application will be ultimately decided by the requirements of the experiments balanced by the complexity one is willing to handle. We aim to illustrate a suite of possibilities and inspire future applications.

\section{Applications and design considerations of worm-chips}

Many different types of studies with $C$. elegans can be carried out in microfluidic devices. The design of each device is highly dependent on the objective of the experiment to be performed. Chips used for C. elegans studies can broadly be divided into those dealing with behavioral assays, high-resolution imaging, and on-chip culture, although no device falls into a single category. Most devices are PDMS chips with channels, chambers or pillars. The majority of these devices are bonded to a glass slide and are thus closed to the environment, as the example shown 
in Figure 1A. These types of devices contain access ports for worm entry and exit. The channels and chambers are filled with liquid media, typically M9 buffer, and access ports allow the exchange of media thus enabling delivery of soluble chemical stimuli. PDMS chips can also be used on agar surfaces, as the example shown in Figure 1B. In this case, worms crawl on the agar surface while in the presence of a controlled gaseous environment. Typically, these devices are better suited when behavior of worms crawling in agar surfaces or feeding from a bacterial lawn is a must. Recently, however, glass-bonded chips that allow crawling and feeding have also been developed. Glass-bonded devices have the added advantage of being able to incorporate on-chip valves since channels can be pressurized without leakage due to the strong, irreversible bond between PDMS and glass. There is a wide variety of possibilities to incorporate into worm chips, and as previously mentioned, no single chip is identical. The selection of features and degree of complexity to incorporate will depend on the experiment under consideration. Operation of worm chips can be as easy as driving fluid by applying pressure by hand (Chung et al., 2011c; Lockery et al., 2008) while some require syringe pumps or even extensive off-chip support (Chung et al., 2008). It is important to mention that very simple and elegant devices can be used to carry out very interesting experiments with highly relevant scientific output. In the following text, the main applications of worm chips developed thus far are briefly reviewed.

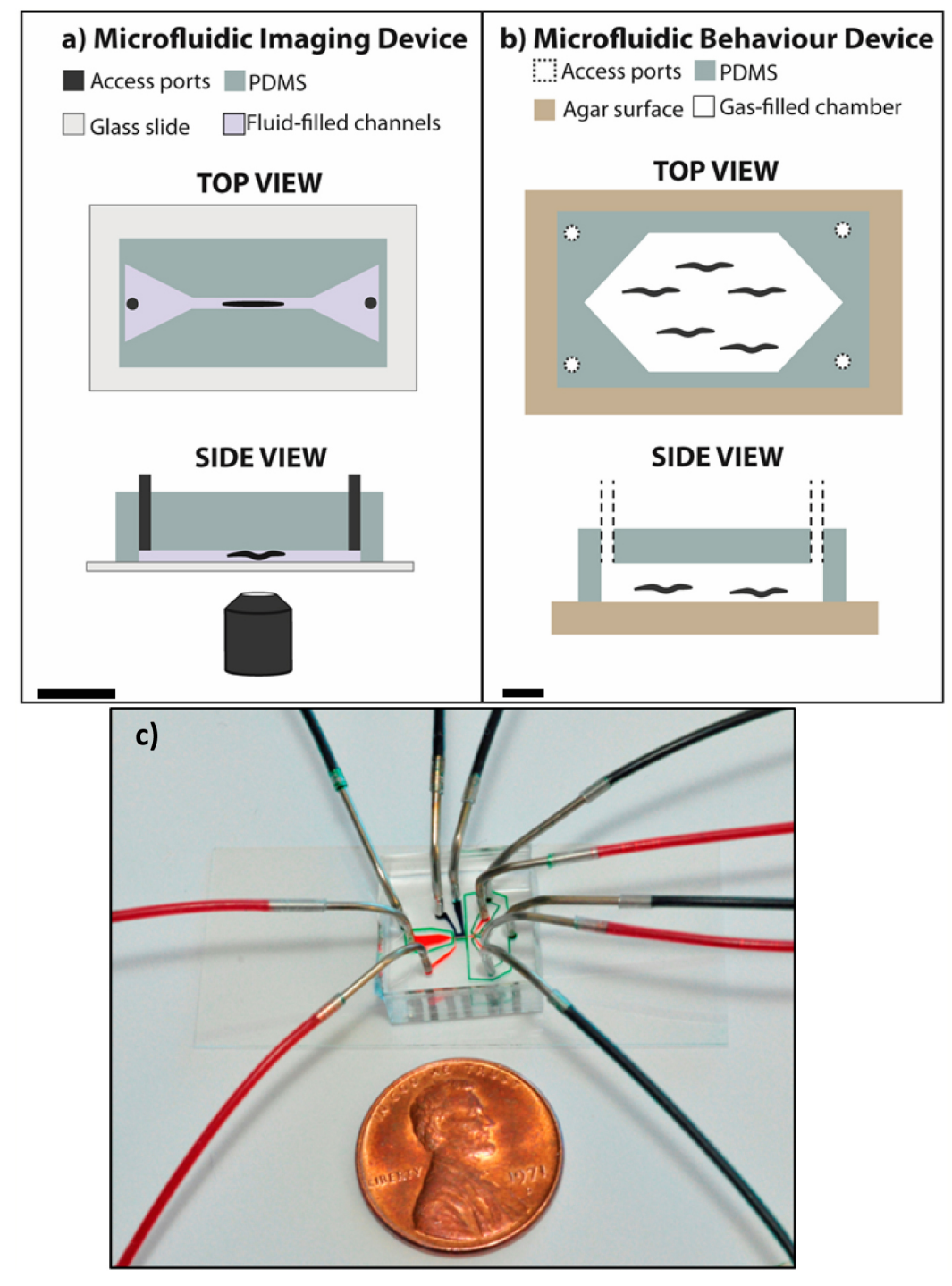

Figure 1. Diagrams representing typical microfluidic devices for C. elegans experiments. A) An example of a PDMS microfluidic device bonded to a glass slide, where worms are imaged in a fluid-filled channel. Access ports allow fluid exchange, worm entry and exit. Worms in imaging devices are typically immobilized by a variety of methods (Section 2.2). B) An example of a PDMS microfluidic device on top of an agar surface to study crawling worms' behavior in gas-filled chambers. Access ports allow gas exchange for stimuli input and controlled gaseous concentrations. Scale bars are $\sim 1 \mathrm{~cm}$ to show typical chip dimensions. C) A microfluidic chip for C. elegans imaging and sorting experiments showing connections to inlet and outlet lines with syringe needle pins and polyethylene tubing. 


\subsection{Animal handling and manipulation}

The ease of worm handling provided by microfluidic devices is one of its most attractive advantages. Devices can be applied to study individual worms or worm populations (Chronis, 2010). In most cases, worms are easily loaded into devices directly by injecting a worm suspension in buffer media. For single-worm imaging studies, worm chips typically contain a wider loading channel or chamber upstream of the imaging channel (Chronis et al., 2007; Stirman et al., 2010). The pressure drop created by flowing the suspension through the device directs the worms towards imaging channels or chambers, where worms can be individually isolated with the inclusion of on-chip valves (Crane et al., 2009; Hulme et al., 2010). The presence of pillars in the loading area upstream of imaging channels has been utilized to bias worm loading towards a head-first orientation (Chokshi et al., 2010), which is useful to avoid stage movement when imaging several worms in series.

The inclusion of channels close to the size of the worm body is desirable in many cases, as it allows high-resolution imaging of neuronal activity in live C. elegans (Lockery, 2007) by restricting its movement. Imaging channels on the scale of the worm body size provide an added benefit for loading: when a worm enters a channel, the fluidic resistance is increased, and thus additional worms are prevented from entering an already loaded channel (Chung et al., 2008). Load regulating valves can also accomplish this objective by isolating worms in the imaging channel (Chung et al., 2008). Some chips contain parallel channels to study several worms at once, as those shown in Figure 2. In this case, the fluidic resistance change diverts incoming worms to other unloaded channels (Hulme et al., 2007; Stirman et al., 2010). In the case of parallel channels, bifurcations can be used so that downstream branches have the same fluidic resistance with no bias in worm distribution (Hulme et al., 2007), as shown in Figure $2 \mathrm{~A}$.

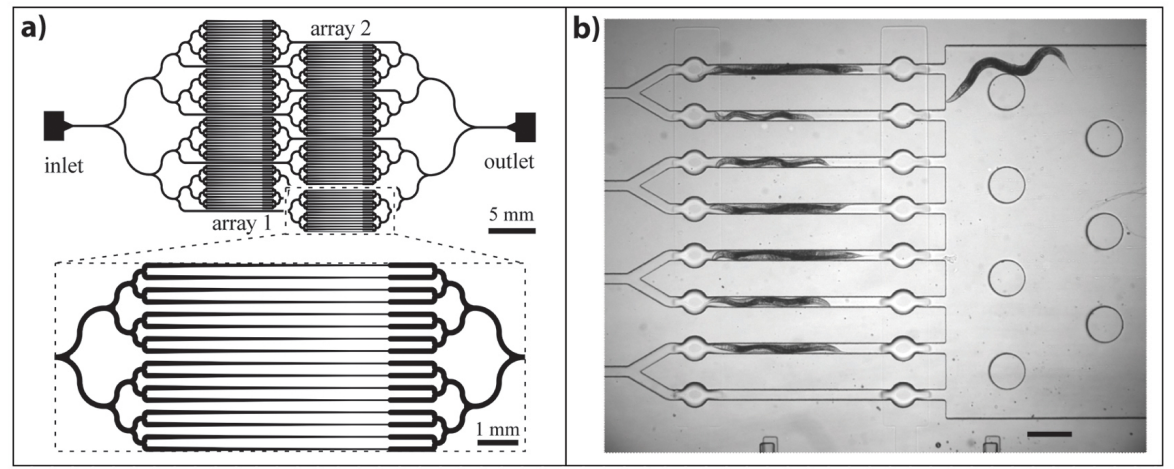

Figure 2. Designs that take advantage of pressure resistance for loading multiple samples in a parallel architecture. Worms that enter a channel increase its fluidic resistance, thus directing the next worms to adjacent channels. A) Microfluidic device with branched network of channels for imaging in worm clamps. Black lines represent channels for worm loading. (Hulme et al., 2007) Reproduced by permission of The Royal Society of Chemistry. B) Microfluidic device for parallel imaging of worms under light stimulation. Worms are studied in channels; area to the right is an unloading chamber. Scale bar is $250 \mu \mathrm{m}$ (Stirman et al., 2010) Reprinted with permission from Elsevier.

\subsection{Worm immobilization and imaging}

Many chips have been devoted to the immobilization of worms in order to allow high-resolution microscopic imaging on chip without the use of anesthetics or glues. Microfluidic devices that are made with a glass coverslip on the bottom are compatible with standard fluorescence microscopy techniques, which allow live imaging of sub-cellular processes. In this instance, immobilization of the worms is necessary. On-chip worm immobilization has been accomplished by cooling (Chung et al., 2008), compression or restriction (Chokshi et al., 2009; Hulme et al., 2007), $\mathrm{CO}_{2}$ (Chokshi et al., 2009), and gelation of the surrounding fluid (Krajniak and Lu, 2010). Each technique confers different advantages and the choice of which one to use depends on the requirements and complexity of the problem and the availability of hardware and software needed to implement them.

Immobilization by compression takes advantage of the flexibility of PDMS, which readily deforms upon exertion of pressure, as shown in Figure 3A. Compression immobilization chips are 2-layer devices that operate in the same manner as on-chip valves. The bottom layer contains a channel where worms are injected, and above it a control layer contains a wider channel that, when pressurized, deforms the membrane (typically 20-75 $\mu \mathrm{m}$ ) between the two layers (Unger et al., 2000). The deflected membrane pushes the worm towards one side of the channel and 
against the glass slide, thus immobilizing it. It is important that the top channel is wide enough to allow sufficient membrane deflection (Chokshi et al., 2009; Gilleland et al., 2010). Additionally, a side suction channel can be included to help position the worm at a certain point along the channel (Zeng et al., 2008).

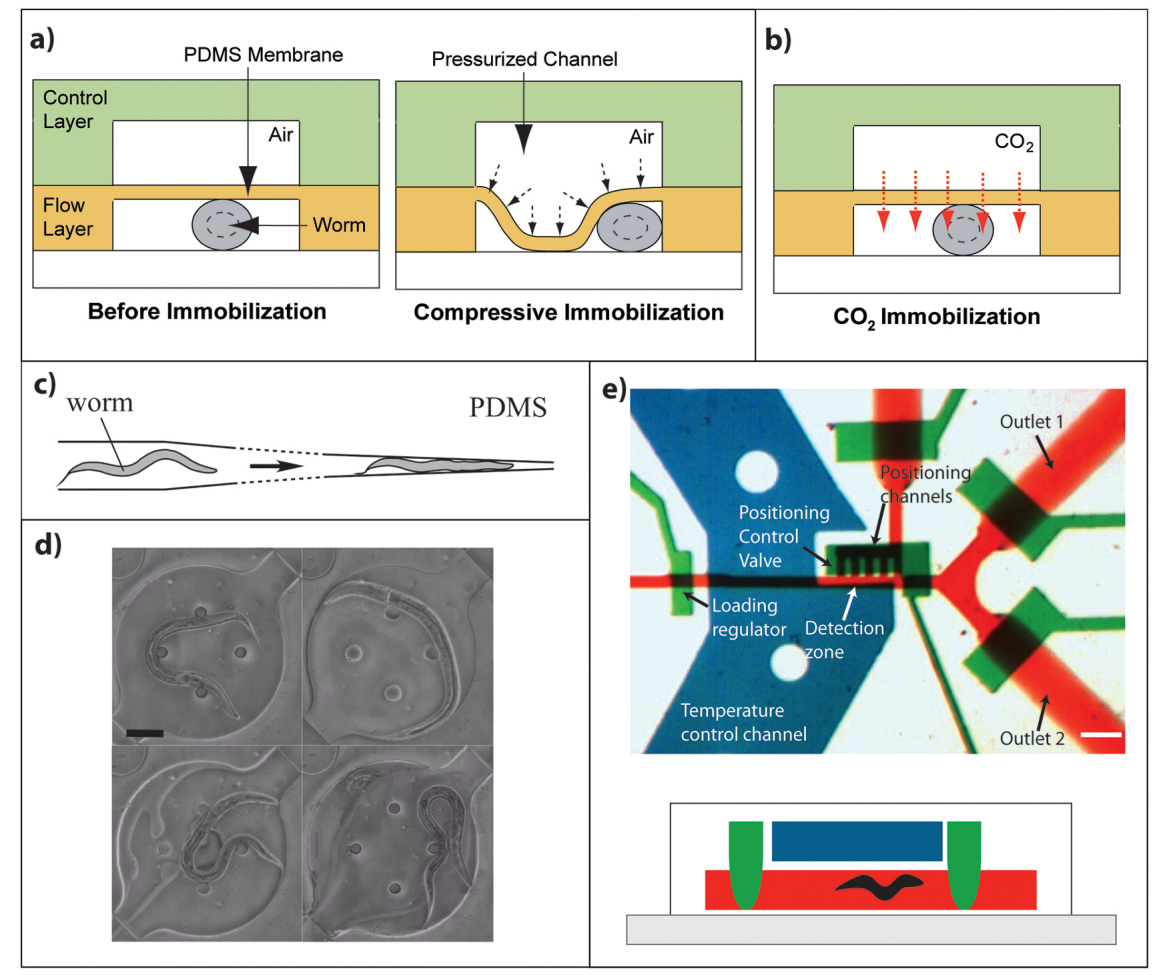

Figure 3. Worm immobilization techniques in microfluidic devices. A) Physical immobilization: compression of a membrane deflected by pressure in the control channel. The yellow layer represents a PDMS layer containing the channel for worm imaging. The green layer is a second PDMS layer on top of the worm imaging with an air-filled channel that when pressurized deflects the PDMS membrane for worm immobilization. The bottom white represents a glass slide.(Chokshi et al., 2009) Reproduced by permission of The Royal Society of Chemistry. B) Chemical immobilization: CO diffusion through adjacent channel (Chokshi et al., 2009) Reproduced by permission of The Royal Society of Chemistry. C) Physical restriction in worm clamp, top view of device design. (Hulme et al., 2007) Reproduced by permission of The Royal Society of Chemistry. D) Physical constraint gel, worm is immobilized by gelation of surrounding fluid at physiological temperatures; scale bar is $60 \mu \mathrm{m}$ (Krajniak and Lu, 2010) Reproduced by permission of The Royal Society of Chemistry. E) Cooling. Top: top view of a high-throughput imaging and sorting device that applies flow of a cooling fluid in a channel (blue) on top of the worm imaging channel (red). Green channels show valves to stop flow and worms; scale bar is $100 \mu \mathrm{m}$ (Chung et al., 2008) Reprinted by permission from Macmillan Publishers Ltd: Nature Methods, copyright 2008. Bottom shows a side view of the same device with green valves closed.

The pressure required to deflect the membrane enough to fully immobilize worms will depend on the membrane geometry, thickness, and stiffness of PDMS. For a 20:1 monomer to cross-linker ratio, a $30 \mu \mathrm{m}$ membrane requires 25 psi for worm immobilization (Chokshi et al., 2009). This pressure has been shown to be appropriate for short immobilization periods without visible damage to the worms. However, for extended immobilization times or to completely impede movement, as required for imaging of sub-cellular processes, other techniques may be better suited.

Worm clamps, also called traps, are another immobilization technique based on physical restriction (Hulme et al., 2007). In this case a channel larger than the worm's diameter is tapered to an opening of $20 \mu \mathrm{m}$ or less (for adult worms) as shown in Figure 3C. Worms are trapped in the resulting wedge-shaped channel. Trapped worms can then be released by reversing the flow or pressurizing the channel. Worm traps, which allow movement in one plane but restrict it in the $z$ direction, have also been used to study worm locomotion and relate it to neuronal activity using calcium imaging (Chronis et al., 2007). In this case, immobilization does not need to be perfect since movement can be corrected by imaging software. Recently, a study that combines calcium imaging and optogenetics in a simple microfluidic device that restricts worms by the mid-body section has been applied to study coordinated motor neuron activity in C. elegans driving forward locomotion (Wen et al., 2012). Other applications that involve calcium imaging on-chip are discussed in the following section (Section 2.3).

Neither compression nor worm traps stop pharyngeal pumping and muscle contractions, which can potentially affect imaging of high-resolution sub-cellular processes. In contrast, immobilization by cooling does stop all of the worm's internal movements. This type of immobilization works by flowing a cooling fluid through a separate flow 
channel adjacent to the worm channel. Efficient heat transfer rapidly cools the worm in the channel to $\sim 4{ }^{\circ} \mathrm{C}$ and consequently immobilizes it (Figure 3E). In this way, sub-cellular processes, such as synapses, can be imaged at high magnification (Chung et al., 2008).

Another recently developed method involves the injection of a Pluronic ${ }^{\circledR}$ F127 solution to worm-loaded chambers. This biocompatible solution reversibly gels upon a very slight temperature increase $\left(\sim 2{ }^{\circ} \mathrm{C}\right)$ and thus immobilizes the worms (Krajniak and Lu, 2010) as shown in Figure 3D. Additionally, worms can be immobilized by flowing $\mathrm{CO}_{2}$ in a channel adjacent to the worm channel (Figure 3B). $\mathrm{CO}_{2}$ diffuses through the PDMS membrane into the fluid surrounding the worm whereby the worm is immobilized; this method can be used for as long as 1-2 hrs of immobilization (Chokshi et al., 2009).

The design and scheme to select would depend on the requirement of the specific application. When imaging very small features, absolute immobilization is necessary. When sorting after imaging is desired, the device design must take into consideration flow control in a single direction, along with the features required for immobilization. Functional imaging would require imaging near room temperature. Finally, all designs should ensure that the selected immobilization scheme (whether it is temperature, carbon dioxide or pressure) does not significantly alter the biological features of interest or process under study.

\subsection{Behavioral assays}

A large number of worm chips have been used to study behavioral and cellular responses of worms to controlled stimuli. These include spatial and temporal, gradient, and step stimuli. Some examples include the responses to dissolved gas concentration, nutrients, chemoattractants, pheromones, pathogenic substances, and temperature (Albrecht and Bargmann, 2011; Chalasani et al., 2007; Chokshi et al., 2010; Chronis et al., 2007; Gray et al., 2004; McCormick et al., 2011; McGrath et al., 2009; Qin and Wheeler, 2007; Zhang et al., 2005). The first example of controlled chemical stimulus showed the generation of a spatial linear oxygen gradient in a worm chamber (Gray et al., 2004). Such spatial, as well as temporal concentration changes, can be generated by flowing gases through channels adjacent to the worm channel (Figure 4A). Gas-phase stimuli have also been applied on single worms by creating temporal step changes in the oxygen or $\mathrm{CO}_{2}$ concentration (Zimmer et al., 2009), where neuronal activities can be recorded and correlated (Figure 4B). This type of gaseous chemical stimuli has also been accomplished by creating a laminar gaseous flow over a worm arena (Bretscher et al., 2008; McGrath et al., 2009). 


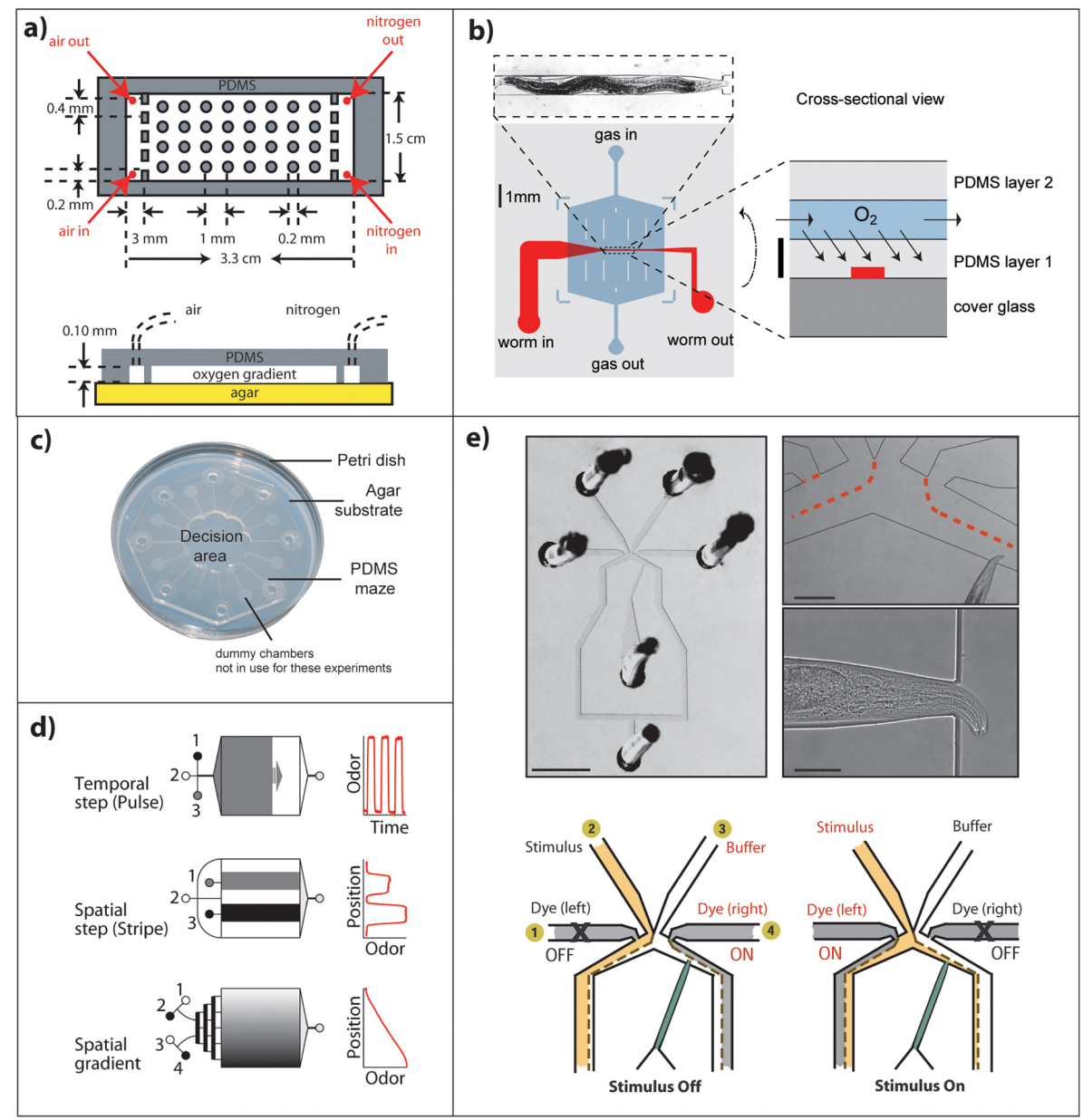

Figure 4. Chips for controlled stimulus delivery. A) Oxygen gradient in aerotaxis microfluidic device. Top: top view, bottom: side view. (Gray et al., 2004) Reprinted by permission from Macmillan Publishers Ltd: Nature, copyright 2004. B) Microfluidic chip for temporal delivery of oxygen, scale bar of cross-section is $100 \mu \mathrm{m}$. (Zimmer et al., 2009) Reprinted with permission from Elsevier. C) Olfactory maze for olfactory preference investigation. Maze width is $\sim 5 \mathrm{~cm}$. Worms are located between the agar surface and the PDMS maze, and can thus access directly test chambers containing bacterial lawns. (Zhang et al., 2005) Reprinted by permission from Macmillan Publishers Ltd: Nature, copyright 2005. D) Designs of microfluidic chips for different types of stimulus delivery to a worm arena. Arena is a chamber $20 \mathrm{~mm}$ in length. This area is a pillar-filled microfabricated PDMS chip bonded to a glass slide, which allows worms to crawl in the fluid-filled chamber. Profiles of the stimulus that can be generated with each design are shown to the right. (Albrecht and Bargmann, 2011) Reprinted by permission from Macmillan Publishers Ltd: Nature Methods, copyright 2011. E) Olfactory chip for delivery of chemicals to the worm nose. Top shows micrographs of chip with a worm loaded and its nose protruding to the stimulus injection region. Bottom shows the control of stimulus injection by alternating with buffer injection. Scale bars: upper left image, $2 \mathrm{~mm}$; right images, $150 \mu \mathrm{m}$. (Chronis et al., 2007) Reprinted by permission from Macmillan Publishers Ltd: Nature Methods, copyright 2007.

Microfluidic devices have also been applied to provide worms with choices between four different odors in an eight-arm maze by positioning a microfluidic maze on top of an agar surface. The preference of trained worms to food odors over pathogenic bacteria odors was observed in this device (Zhang et al., 2005) (Figure 4C). Another device that has been successfully applied for chemical stimuli input is the olfactory chip (Chalasani et al., 2007; Chokshi et al., 2010; Chronis et al., 2007). This device contains a worm trap that allows the nose to protrude into a channel where a chemical stimulus is delivered (Figure 4E). The worm trap allows high-resolution imaging of neuronal activity by calcium imaging. Four channels are used for injection of stimulus and buffer or dye, which allows easy visualization and helps to avoid fluidic disruptions.

The effect of chemical stimuli on the movement responses of freely moving or semi-restrained worms can also be made possible with microfluidic chips. In one example, worms subject to a chemoattractant stimulus are held at the mid body section by two adjacent suction channels while the stimulus is delivered to the freely-moving anterior part of the worm (McCormick et al., 2011) and the subsequent head position response is scored. Chemical stimuli applied to freely moving worms can be generated as temporal pulses, gradients, and spatial bands by simply 
including inlet channels with different designs (Albrecht and Bargmann, 2011) (Figure 4D). These types of stimuli have been applied to study locomotion of worm populations in a pillar-filled arena, where the pillars are designed such that they allow crawling-like behavior even though animals are immersed in liquid environments (Figure 5A).

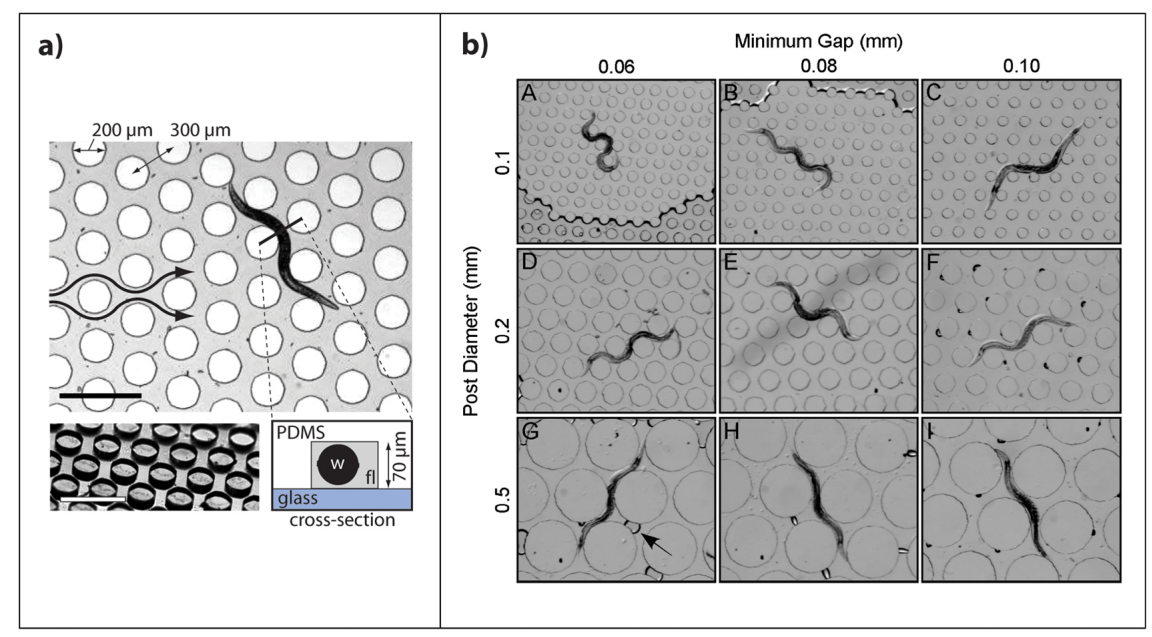

Figure 5. Inclusion of physical structures to enhance or study locomotion on-chip. A) Worms crawling in micro-post filled arena under chemical stimuli, scale bars are $500 \mu \mathrm{m}$. (Albrecht and Bargmann, 2011). Reprinted by permission from Macmillan Publishers Ltd: Nature Methods, copyright 2011. B) Worm crawling behavior in artificial dirt chips with varying feature size and spacing. (Lockery et al., 2008). Reproduced with permission of the American Physiological Society.

Other chips have also taken advantage of the micro-scale features that can be generated by soft lithography to construct patterned environments and thus study the worm's movement and responses to them. The "artificial dirt" chip (Figure 5B) is a device consisting of arranged pillars at different spacing to mimic dirt that nematodes encounter in nature (Lockery et al., 2008). This type of study has also been performed in an agar device constructed from a PDMS mold (Park et al., 2008). The locomotion of worms has also been studied in waveform channels fabricated with different amplitude and wavelength (Lockery et al., 2008). From these studies, it has been found that there are optimum values of amplitude where worms can more efficiently move. Microfabrication has also been used in a force sensing device where SU-8 pillars attached to cantilevers are used to measure the forces exerted by crawling worms causing pillar deflection (Doll et al., 2009).

\subsection{Chambers and droplets}

Studies of freely moving single worms can be performed with microfluidics by either incorporating chambers in the device or utilizing droplets to encapsulate single worms (Carr et al., 2011; Chung et al., 2011c; Clausell-Tormos et al., 2008; Hulme et al., 2010; Kim et al., 2007; Krajniak and Lu, 2010; Shi et al., 2008; Shi et al., 2010). Microfabricated chambers in PDMS devices distribute individual worms to separate compartments. Worms in large chambers can grow without restrictions and experience negligible shear in comparison with smaller channels. Food, odor, tastants, pheromones, and drugs can be supplied by an inlet channel to the chamber, while waste and debris can be removed through an outlet channel. These connections should be small enough to prevent worms from escaping. Additionally, on-chip valves can be used to close chamber inlets and outlets.

Longitudinal observation throughout a worm's culture period in chambers can be performed by incorporating an adjacent worm clamp (Hulme et al., 2010) to trap and image the worm and then releasing it back to the chamber, as shown in Figure 6A. Alternatively, reversible immobilization of worms in the chambers for high-resolution imaging can also been accomplished by introducing a Pluronic F127 solution, which gels upon a slight temperature increase (Krajniak and Lu, 2010). The design of this type of device is shown in Figure 6B. 


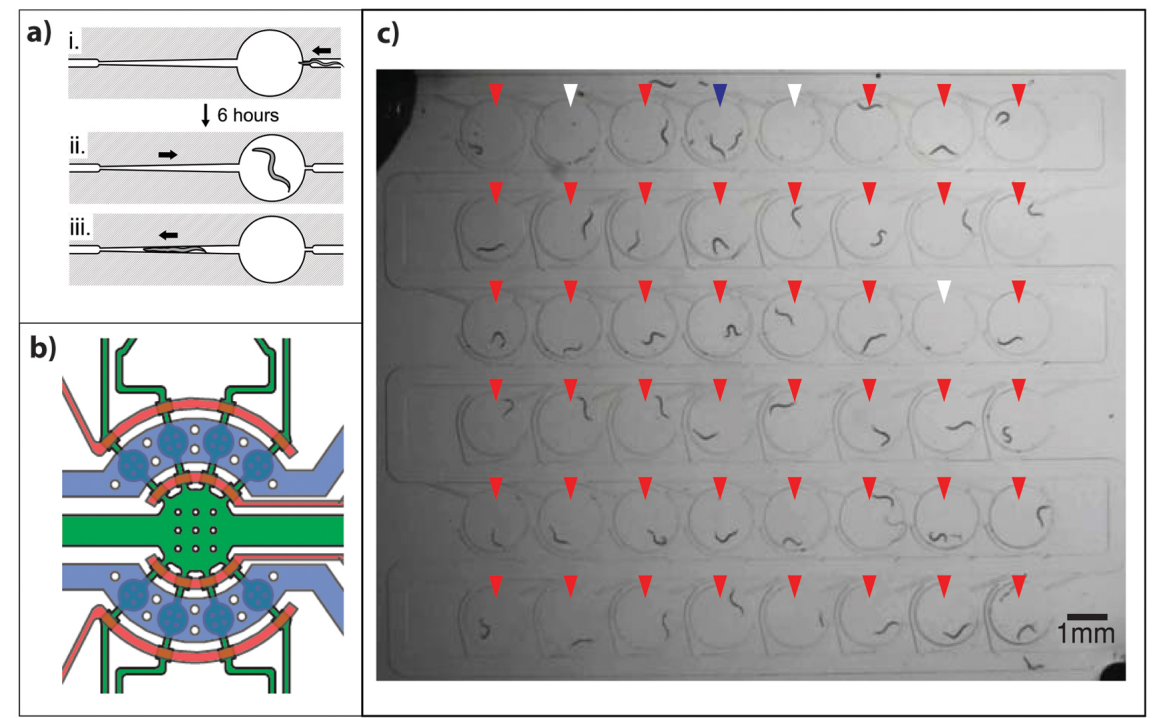

Figure 6. Worm chambers in microfluidic devices. A) Array of chambers for simultaneous observation of multiple worms (Chung et al., 2011c) B) Chip with chambers incorporating an adjacent worm clamp for longitudinal observation (Hulme et al., 2010). C) Worm chambers with immobilization by gelation (Krajniak and Lu, 2010). All images reproduced by permission of The Royal Society of Chemistry.

Recently a device consisting of an array of 48 chambers, which allows the observation of multiple worms at the same time, was generated (Figure 6C). The inlet of each chamber is a very narrow channel, which traps a single worm. Pressurizing the main channel allows expansion of the entry ports thus loading a single worm into each chamber. Chemical stimuli can also be delivered to worms in these chambers and the worm's behavioral response while freely swimming can be studied (Chung et al., 2011c).

Lifespan analysis has also been performed in a single-animal culture multiwell apparatus made of a perforated glass slide sealed by a PDMS membrane. Each well containing NGM and E. coli provides a chamber for lifelong cultivation and analysis of individual worms (Pincus et al., 2011). In addition to culturing, development, and behavioral analysis, chambers have also been used as drug reservoirs for worm exposure. In this application, a worm is loaded into the drug reservoir for a given time and its behavior is subsequently monitored in an adjacent observation channel (Carr et al., 2011). Chambers have also been included in a compact disc device developed to culture worms under high gravity conditions (Kim et al., 2007).

A different approach to trap individual worms in separate compartments has focused on encapsulating worms in droplets. The controlled flows obtained in microfluidic devices can be manipulated to generate water-in-oil droplets with uniform size. Worm eggs have been encapsulated in water droplets surrounded by silicon oil, after which the larva hatches inside the droplet (Clausell-Tormos et al., 2008). Water droplets generated in microfluidic devices can be then trapped in separate compartments inside the device (Shi et al., 2008). Droplets can also be squeezed into worm traps for imaging of high resolution processes (Shi et al., 2010). Although this technique is an excellent approach for individual worm studies, especially for very young worms otherwise difficult to isolate individually, some challenges include proper feeding of the animals and the presence of surfactants that may alter both the experimental conditions and the physiology. In this scenario, proper controls would need to be conducted.

\subsection{High-throughput imaging and screening}

One of the most important applications of microfluidics has been high-throughput automated imaging and screening of worm populations, as it can greatly reduce the time required to study each worm. These chips are intended to screen for interesting mutants, drugs or RNAi libraries. In general, these chips contain an imaging channel and two outlets for worm sorting and recovery, as shown in Figure 3E. Devices that have been successfully used to identify interesting mutants incorporate a cooling channel for worm immobilization as well as a channel for positioning which guides the worm into the desired location for imaging (Chung et al., 2008; Lee et al., 2013). Interfacing these devices with image processing software allows for easier mutant identification (Crane et al., 2009; Lee et al., 2013). A device for screening and sorting animals, which contains a side suction channel to hold the worm in place while imaging and then releases it towards one exit or the other, has also been developed (Rohde et al., 2007). 
Additionally, high-throughput imaging of synaptic transmission has been accomplished by combining microfluidics and optogenetics, a technique to study neuronal function and synaptic transmission whereby specific neurons can be stimulated with light (Stirman et al., 2010). This chip was developed to monitor function of motorneurons, which innervate muscle walls, and when stimulated affect the worm length. This device is high throughput as it allows parallel investigation of several worms on the order of $\sim 100$ animals in less than 5 minutes. Other experiments, such as drug or RNAi screens, can also be performed using such a setup as long as the readout is morphometric (either gross change of the body or that of a fluorescent marker).

A microfluidic device has also been recently applied for whole-animal drug screening with electrophysiological measurements. This device consists of parallel worm channels with a worm trap and electrodes that detect electrical activity and thus measure neuromuscular function (pharyngeal pumping specifically). Delivery of test solutions is possible and resulting changes in electrophysiological activity with high signal-to-noise ratio can be recorded (Lockery et al., 2012).

\subsection{Laser microsurgery}

Performing on-chip laser ablation has been successfully achieved to study neuronal function and axonal regeneration (Ben-Yakar and Bourgeois, 2009). Axonal regeneration studies have been carried out on-chip through femtosecond laser nanoaxotomy while applying compression immobilization. Adjacent recovery chambers for worm feeding allow monitoring axonal regeneration. These studies found that regeneration occurs much faster if no anesthetics are used (Guo et al., 2008), evidencing the advantage of drug-free immobilization in microfluidic devices.

A device to perform axotomy in animals exposed to a chemical library in multiwell plates has also been developed. In this case worms are held by suction channels and a compression valve while laser ablation of axons takes place in user-defined points (Samara et al., 2010). Immobilization of worms in a clamp has been proven useful to perform single synapse ablation in microfluidic devices (Allen et al., 2008). More recently, a high-throughput device for on-chip laser ablation of cells in a worm immobilized through cooling has been accomplished (Chung and $\mathrm{Lu}, 2009$ ). This device is interfaced with computer software for image processing, which allows automated cell recognition and ablation. Furthermore, the device incorporates two sets of worm loading channels, which can operate in parallel for continuous operation.

\section{Resources for chip fabrication}

Fabrication of microfluidic devices with soft lithography is a simple and inexpensive process. However, setting up chip microfabrication capabilities in-house involves purchasing and installing equipment that will require maintenance and troubleshooting. For laboratories that do not intend to be fabrication-intensive, there are publicly available resources that provide microfluidic device fabrication services. The Stanford microfluidics foundry (http://www.stanford.edu/group/foundry/), managed by Stephen Quake's lab, and the Caltech's foundry (http://kni.caltech.edu/foundry/), for example, can generate both masters and microfluidic devices from a CAD design. These are resources to consider especially if devices will not be frequently used, no or little iteration/alteration of design is anticipated, or devices can be re-used after cleaning. In this way, the time and costs of installing, maintaining, and troubleshooting equipment can be circumvented. Ultimately, in deciding whether to make microfluidic devices in-house or utilize public resources, one should consider the frequency and volume of chips utilized, whether new designs are being considered, as well as external fabrication costs versus equipment setup, maintenance costs, and time.

\section{Fabrication of microfluidic devices for $C$. elegans studies with soft lithography in-house.}

Fabrication of PDMS microfluidic devices by soft-lithography rapid prototyping is simple, inexpensive, and fast (Duffy et al., 1998; McDonald et al., 2000). The complete process can be finished in one day, while molding of PDMS devices takes only a few hours. Soft lithography (Xia and Whitesides, 1998) is based on the concept of cast molding. The basic concept relies on the generation of a master mold with the desired (negative) features in high-relief. A liquid silicon pre-polymer mixture is then poured onto the mold and cured until solidified. The solid elastomer with the desired features is then peeled-off. The process is shown in Figure 7 and explained in detail next. 


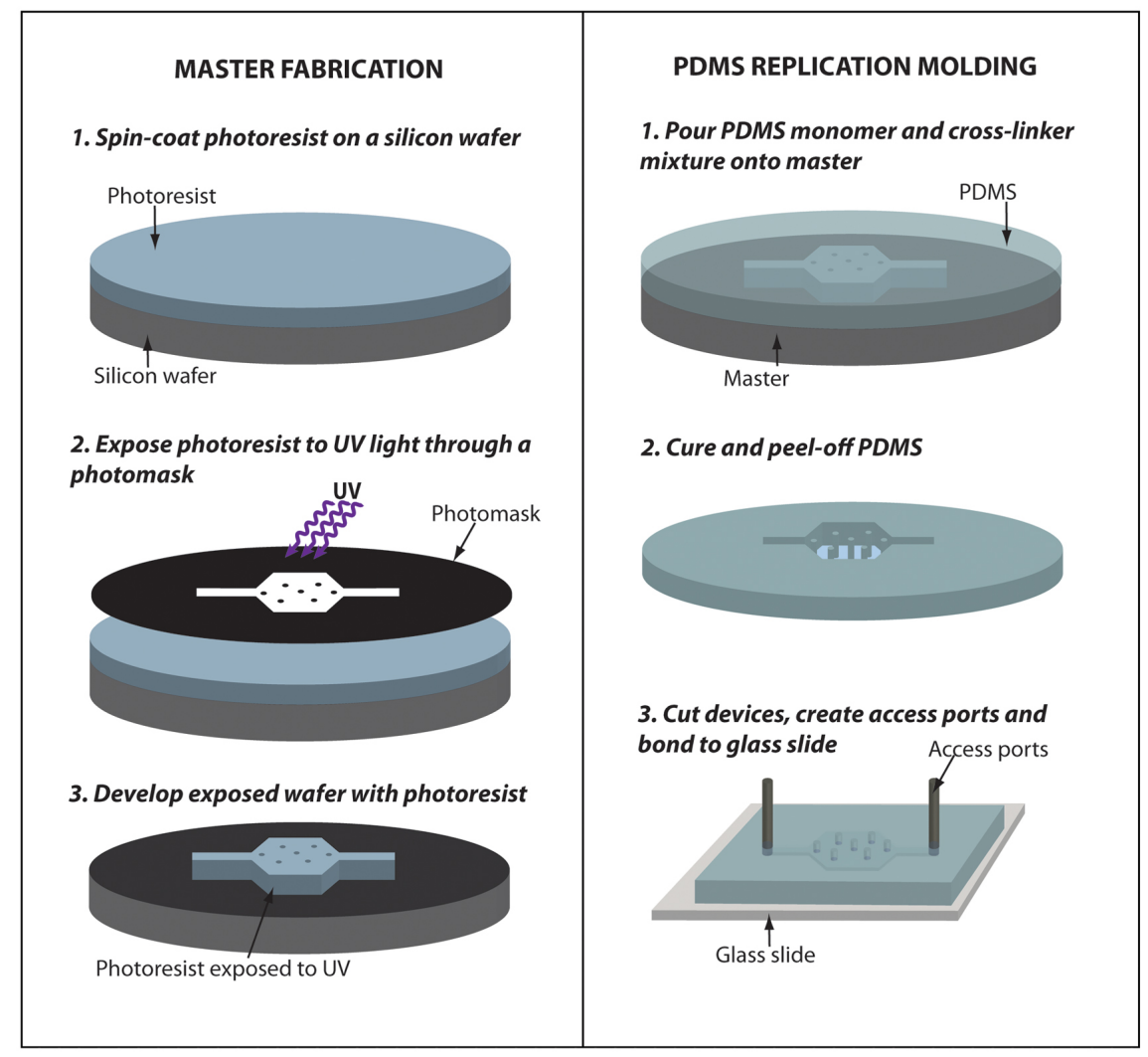

Figure 7. Fabrication of microfluidic devices with PDMS replica molding. The master fabrication steps are typically performed in a cleanroom environment although it is also possible outside if features of interest are large; the replica steps are typically done on lab benches.

\subsection{Device design and masters}

The first step in fabrication is the device design, which is typically generated in a computer-assisted design program (CAD) (e.g., AutoCAD ${ }^{\mathbb{R}}$ software) or other drawing programs (e.g., Adobe ${ }^{\circledR}$ Illustrator $^{\mathbb{R}}$ ). In this step, several design considerations need to be taken into account. The size of the channels should be determined by both their function and the size of the worms to be used. Curved, straight, and angled features can be included, but the minimum feature size attainable should be considered. High-resolution printing allows obtaining features with up to $\sim 8 \mu \mathrm{m}$ lateral resolution (Sia and Whitesides, 2003). Additionally, the aspect ratio of the device should not fall below 1:10 (height to width); inexperienced users should aim for even smaller ratios. If wider channels are required, posts can be included to support the ceiling of the structure so it does not collapse.

The physical masks are then fabricated on chromium-on-quartz plates (by a mask-making company) or printed on a flexible transparency. For designs with features smaller than $8 \mu \mathrm{m}$, chrome masks are recommended. Most cleanrooms offer mask fabricating/printing services; additionally, this service is offered by several companies such as CAD/Art Services, Inc., Artnet Pro Inc., and Infinite Graphics. The transparency is then used as a mask for exposure of photoresist to UV light only in designated areas. UV light passes only through clear regions in the mask onto the photoresist, which has been spin-coated on a silicon wafer. Exposure of photoresist to UV light crosslinks (negative photoresists) or degrades (positive photoresists) the exposed areas. The photomask can be printed clear-field or dark-field, depending on the photoresist to be used. The most commonly used is SU-8 from MicroChem, a negative photoresist, with a dark-field mask. However, a good reason to use positive photoresist is that they can reflow when baked at high temperature (Unger et al., 2000). In this way, initially square channels can become round, as shown in Figure 8. Round channels are useful in the case where fully closable valves are required (as explained in Section 4.3). Once the photoresist is exposed, it is developed with photoresist-specific solvents that remove un-crosslinked polymers. Specific developing and baking protocols for each photoresist are available through the manufacturers. 


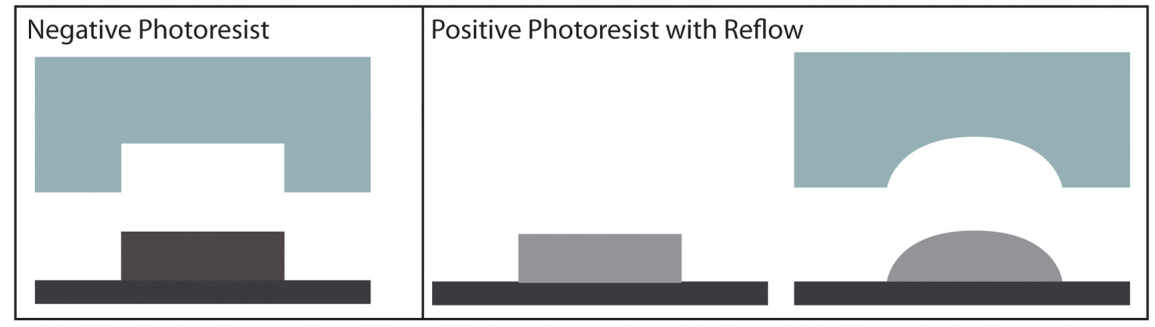

Figure 8. Cross-sectional views of square and rounded channels with negative and positive photoresist, respectively. Rounded channels can be closed off by a valve membrane, while square channels can act like a sieve, both useful for different applications.

The height of the master features is obtained by the thickness of the spin-coated photoresists, which depends on the photoresist viscosity and spinning velocity. Typically, heights of 1-300 $\mu \mathrm{m}$ can be achieved with SU-8. Positive resists can also give thin $(\sim 1 \mu \mathrm{m})$ to thick $(\sim 20-100 \mu \mathrm{m})$ layers. One should always check with manufacturers to confirm the performances of the photoresists. Additionally, masters with different height levels can be generated by spin-coating, exposing, and developing a second photoresist layer onto a previously fabricated first layer (Anderson et al., 2000).

Once the master is fabricated, it is typically treated with fluorinated silanes to prevent irreversible adhesion between the master and PDMS (Duffy et al., 1998). A detailed protocol for simple single layer master molds is included next.

\subsubsection{Master mold fabrication protocol for single layer devices:}

\section{Required equipment and supplies:}

- Silicon wafers

- Wafer tweezers

- Piranha etch bath and etch solution

- SU-8 (MicroChem)

- Transparency mask

- SU-8 developer (MicroChem)

- Isopropyl alcohol

- Deionized water

- Nitrogen for drying

- Silanizing agent

- 2 (or 3) hot plates

- Spin-coater: Equipment used to create a uniform layer of a specific height of un-crosslinked photoresist on a silicon wafer by spinning the substrate (e.g., WS-650Hz-23 Spin Coater from Laurell Technologies Corp. ${ }^{\mathbb{B}}$ )

- Mask-aligner (with $365 \mathrm{~nm}$ UV light source): Equipment used to cross-link the photoresist by exposing the photoresist through the aligned mask to UV light (e.g., Karl Suss MA6)

- Vacuum pump and desiccator

- Mask (printed on emulsion films) 
1. Cleaning silicon wafers: Prior to processing, it is important to ensure silicon wafers are clean. This is typically accomplished by placing the wafers in a bubbling piranha solution (1:3 sulfuric acid and hydrogen peroxide) for 10 minutes. Caution: the mixing process is strongly exothermic and releases hot vapor, and the mixture is highly corrosive and oxidating. This step should be performed with extreme care and with personal protective equipment including face shield, apron, and gloves. Afterwards, the wafers are rinsed with deionized water. Cleaned wafers are then dried with nitrogen gas and can be stored in a clean box prior to use.

2. Dehydrate clean wafers: Set a clean wafer on a hotplate at $200{ }^{\circ} \mathrm{C}$ for 15 minutes to evaporate any residual humidity. Allow the wafer to cool to room temperature before proceeding to the next step.

3. Spin-coat photoresist: According to the desired height of the device, spin-coat the photoresist onto the wafer. Check the photoresist manufacturer's instruction for speed, acceleration, and time that will result in the desired coat thickness.

4. Soft-bake: Bake the spin-coated wafer on a hotplate at $65{ }^{\circ} \mathrm{C}$ for the amount of time specified in the manufacturer's instruction for coatings of your desired thickness. Allow to cool to room temperature before proceeding to the next step.

5. Exposure: Measure the intensity of the light provided by the mask aligner. According to the thickness of your coating, a certain exposure energy is required (see the manufacturer's instructions). Calculate the exposure time required with this information. When exposing, hard contact is recommended and making sure the ink side of the transparency is in contact with the wafer. (The mask should be printed emulsion down and used with the emulsion side in contact with the photoresist to ensure fidelity).

6. Post exposure bake: After exposure, bake the wafer on a hotplate at $95{ }^{\circ} \mathrm{C}$ for the amount of time specified for your device height. Be sure to level the hotplate. Allow the wafer to cool to room temperature before proceeding.

7. Development: Immerse the wafer in a container with photoresist developer; gently move the container around to mix the solution. The developing time will depend on the features as well as the film thickness. Consult manufacturer's instructions. After the completion of the development, rinse the wafer with isopropyl alcohol and then deionized water. Dry with nitrogen gas. Milky streaks on the wafer are indicative of under-development; if this is the case, develop the wafer further as before.

8. Silanization: The wafer should be completely dry for this step. Place your wafer and an open glass vial with a drop or two of the silanizing agent in a desiccator. Apply vacuum for 20 minutes, leave under vacuum for several hours or overnight.

\subsection{PDMS Replication Molding}

The next step in the fabrication process is cast molding. PDMS pre-polymer is mixed with cross-linker and poured onto the mask. Although other elastomeric materials can be used, PDMS provides the benefit of conforming to the master's features even at the micron-scale (Sia and Whitesides, 2003). PDMS elastomer kits are commercially available; the most common are Sylgard ${ }^{\circledR} 184$ (Dow Corning) and RTV 615 (Momentive Performance Materials Inc.).

The cross-linker to pre-polymer ratio defines the stiffness of the PDMS after curing. Typically a 1:10 ratio is used. Devices that require softer features can be poured at 1:20, while stiffer PDMS can be accomplished by reducing this ratio up to 1:5, for example. Before pouring, this mixture is exposed to vacuum to remove air bubbles that can get trapped between the master and PDMS. Once the mixture is degassed it can be poured onto the master and cured at approximately $70{ }^{\circ} \mathrm{C}$ for $\sim 1-2$ hours or overnight at room temperature. After the device is cured it can be easily peeled off the master. A detailed protocol to cast PDMS microfluidic devices from a microfabricated master follows.

\subsubsection{PDMS device replication molding protocol}

\section{Required equipment and supplies:}

- Master mold (silicon wafer with photoresist features) 
- Vacuum pump and desiccator

- Balance

- Stopwatch (timer)

- Oven

- Scalpel and razor blades

- Compressed air or nitrogen

- Scotch tape

- Blunt syringe needles (or biopsy punches)

- Plasma etcher (with vacuum pump): Equipment that generates a plasma from a gas (oxygen in this case) (e.g., Harrick Plasma). Exposure of glass and PDMS surfaces to the plasma removes impurities and creates chemical charged groups on the surface, required for irreversible bonding.

- Plastic petri dishes

- Glass slides or cover-slips

1. PDMS mixing: Weigh the desired amount of PDMS pre-polymer in a disposable cup. Pour the appropriate amount of cross-linker according to the desired final elastomer stiffness (typical is 1:10). Mix the pre-polymer and cross-linker with a plastic spatula.

2. De-gassing: Place the plastic cup in a desiccator under vacuum for approximately 30 minutes or until all the bubbles have disappeared.

3. PDMS casting: Place the master in a plastic petri dish and pour the de-gassed elastomer mixture onto the master. Try to avoid the creation of bubbles in the process. If there are visible bubbles, the poured master can be placed under vacuum until the bubbles disappear. Bake in an oven at $70{ }^{\circ} \mathrm{C}$ for 2 hours. Once the PDMS has cured, gently peel it off of the master. Use a razorblade or scalpel to cut out the devices.

\subsection{Valves and 2-layer devices}

On-chip valves can be fabricated on the devices by adding a second control layer on top of the main flow channels (Unger et al., 2000). Pressurizing the control layer deflects the membrane between both layers, thus closing the flow channels. Channels on the control layer should be designed without an outlet so that they can be easily pressurized. Two-layer devices are generated by utilizing two separate masters. The flow-layer master is spin-coated with PDMS to provide a membrane thickness of $20-50 \mu \mathrm{m}$. Partially cured layers from each master mold are then brought into contact, aligned and allowed to bond and completely cure. Valves fabricated this way can be either partially or fully closable using square or round channels in the flow layer as mentioned earlier (Figure 8). The advantage of these integrated valves is that one can automate the actuations using off-chip components (Chung et al., 2008; Crane et al., 2009).

In addition to these pressurized fluid-actuated valves, simple screw valves can also be incorporated in microfluidic devices (Hulme et al., 2009). These valves contain a screw embedded in the PDMS, which is typically surrounded by a rigid epoxy or polyurethane, as shown in Figure 9. They are easily actuated upon rotation of the screw by hand, which is simple to operate. 


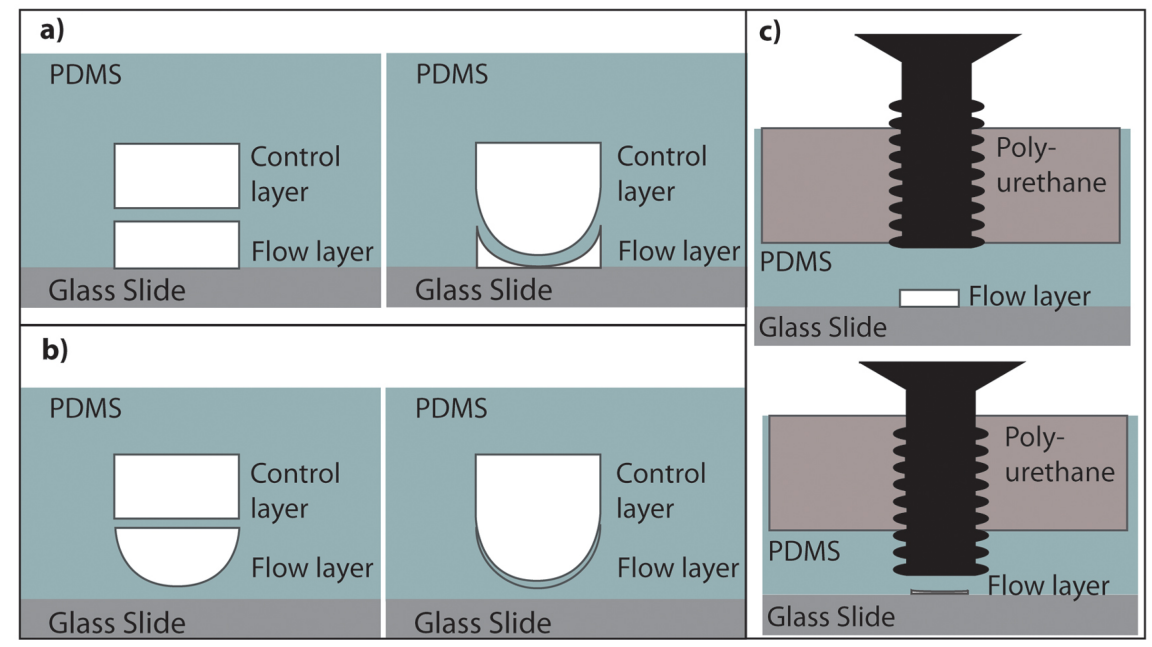

Figure 9. Valves types for microfluidic devices. A) Partial closure valves, B) Full-closure valves, C) Screw valves.

\subsection{Bonding the devices and connections to the macro world}

Access ports to the inlet and outlet of microfluidic devices can be created by cutting holes at the inlet and outlet points in the device. These holes can be made with biopsy punches or sharpened blunt needles of 21 to 19 gauges. It is important to make clean cuts and avoid scraping of PDMS debris, which can clog the devices later on when the devices are in operation. Compressed air and adhesive tape (e.g., Scotch ${ }^{\circledR}$ tape) can be used to remove dust and debris. Afterwards, the devices can be sealed by bonding to a glass microscope slide or coverslip. Irreversible bonding is achieved by treating both surfaces with oxygen or air plasma, which generates silanol groups (Si-OH) on both PDMS and glass surfaces; upon contact a Si-O-Si covalent bond is created (McDonald et al., 2000). The time of plasma activation depends on the power of the plasma generator, the position of the surfaces relative to the plasma, and what gas is used.

Devices are typically connected to inlet and outlet lines through syringe needle pins and polyethylene tubing, as shown in Figure 1C. These connections are inserted in the previously made entry ports. Loading of worm suspensions and delivery of fluids can be performed with the aid of syringe pumps or peristaltic pumps. Connecting inlet ports to sealed pressurized containers is also an effective way to deliver fluids in a controlled fashion, with the aid of pressure regulators (Chokshi et al., 2010; Chung et al., 2008). Worms can also be easily loaded into microfluidic devices by gravity loading (Hulme et al., 2010), whereby placing reservoirs connected to the device at different heights can deliver fluids with different flow rates. Both pressure and gravity delivery systems will not determine flow rates: rather, the flow rates will depend on the pressure resistance in the chip and the external tubing and connectors. The delivery system used will ultimately depend on the specific application, the device design, and the available resources.

\section{Conclusions}

Microfluidics is an easily implementable tool which provides not only a platform for easier worm handling, but also brings about several advantages impossible to realize with typical methods for C. elegans studies, such as imaging high-resolution patterns in un-anesthetized worms. Because of the ease of fabrication and implementation, and all the advantages it provides for worm handling, the applications of this technique for biological studies are expected to increase until it becomes a widespread research tool. Additionally, microfluidics will continue to impact C. elegans research by greatly reducing the typical experimental time lapse. Although many applications of microfluidics have been already shown, there is still a large window of opportunity for developing application-specific chips. 


\section{References}

Albrecht, D.R., and Bargmann, C.I. (2011). High-content behavioral analysis of Caenorhabditis elegans in precise spatiotemporal chemical environments. Nat. Methods 8, 599-605. Abstract Article

Allen, P.B., Sgro, A.E., Chao, D.L., Doepker, B.E., Edgar, J.S., Shen, K., and Chiu, D.T. (2008). Single-synapse ablation and long-term imaging in live C. elegans. J. Neurosci. Methods 173, 20-26. Abstract Article

Anderson, J.R., Chiu, D.T., Jackman, R.J., Cherniavskaya, O., McDonald, J.C., Wu, H.K., Whitesides, S.H., and Whitesides, G.M. (2000). Fabrication of topologically complex three-dimensional microfluidic systems in PDMS by rapid prototyping. Anal. Chem. 72, 3158-3164. Abstract Article

Andersson, H., and van den Berg, A. (2003). Microfluidic devices for cellomics: a review. Sensors and Actuators B: Chemical 92, 315-325. Article

Ben-Yakar, A., and Bourgeois, F. (2009). Ultrafast laser nanosurgery in microfluidics for genome-wide screenings. Curr. Opin. Biotechnol. 20, 100-105. Abstract Article

Ben-Yakar, A., Chronis, N., and Lu, H. (2009). Microfluidics for the analysis of behavior, nerve regeneration, and neural cell biology in C. elegans. Curr. Opin. in Neurobiol. 19, 561-567. Abstract Article

Bretscher, A.J., Busch, K.E., and de Bono, M. (2008). A carbon dioxide avoidance behavior is integrated with responses to ambient oxygen and food in Caenorhabditis elegans. Proc. Natl. Acad. Sci. U. S. A. 105, 8044-8049. Abstract Article

Carr, J.A., Parashar, A., Gibson, R., Robertson, A.P., Martin, R.J., and Pandey, S. (2011). A microfluidic platform for high-sensitivity, real-time drug screening on C. elegans and parasitic nematodes. Lab Chip 11, 2385-2396. Abstract Article

Chalasani, S.H., Chronis, N., Tsunozaki, M., Gray, J.M., Ramot, D., Goodman, M.B., and Bargmann, C.I. (2007). Dissecting a circuit for olfactory behaviour in Caenorhabditis elegans. Nature 450, 63-70. Abstract Article

Chokshi, T.V., Bazopoulou, D., and Chronis, N. (2010). An automated microfluidic platform for calcium imaging of chemosensory neurons in Caenorhabditis elegans. Lab Chip 10, 2758-2763. Abstract Article

Chokshi, T. V., Ben-Yakar, A., and Chronis, N. (2009). $\mathrm{CO}_{2}$ and compressive immobilization of C. elegans on-chip. Lab Chip 9, 151-157. Abstract Article

Chronis, N. (2010). Worm chips : Microtools for C. elegans biology. Lab Chip 10, 432-437. Abstract Article

Chronis, N., Zimmer, M., and Bargmann, C.I. (2007). Microfluidics for in vivo imaging of neuronal and behavioral activity in Caenorhabditis elegans. Nat. Methods 4, 727-731. Abstract Article

Chung, K., Kim, Y., Kanodia, J.S., Gong, E., Shvartsman, S.Y., and Lu, H. (2011a). A microfluidic array for large-scale ordering and orientation of embryos. Nat. Methods 8, 171-176. Abstract Articleu

Chung, K., and Lu, H. (2009). Automated high-throughput cell microsurgery on-chip. Lab Chip 9, 2764-2766. Abstract Article

Chung, K., Rivet, C.A., Kemp, M.L., and Lu, H. (2011b). Imaging single-cell signaling dynamics with a deterministic high-density single-cell trap array. Anal. Chem. 83, 7044-7052. Abstract Article

Chung, K., Zhan, M., Srinivasan, J., Sternberg, P.W., Gong, E., Schroeder, F.C., and Lu, H. (2011c). Microfluidic chamber arrays for whole-organism behavior-based chemical screening. Lab Chip 11, 3689-3697. Abstract Article

Chung, K., Crane, M.M., and Lu, H. (2008). Automated on-chip rapid microscopy, phenotyping and sorting of $C$. elegans. Nat. Methods 5, 637-643. Abstract Article 
Clausell-Tormos, J., Lieber, D., Baret, J.C., El-Harrak, A., Miller, O.J., Frenz, L., Blouwolff, J., Humphry, K. J., Köster, S., Duan, H., et al. (2008). Droplet-based microfluidic platforms for the encapsulation and screening of Mammalian cells and multicellular organisms. Chem. Biol. 15, 427-437. Abstract Article

Crane, M.M., Chung, K., and Lu, H. (2009). Computer-enhanced high-throughput genetic screens of C. elegans in a microfluidic system. Lab Chip 9, 38-40. Abstract Article

Crane, M.M., Chung, K., Stirman, J., and Lu, H. (2010). Microfluidics-enabled phenotyping, imaging, and screening of multicellular organisms. Lab Chip 10, 1509-1517. Abstract Article

Doll, J.C., Harjee, N., Klejwa, N., Kwon, R., Coulthard, S.M., Petzold, B., Goodman, M.B., and Pruitt, B.L. (2009). SU-8 force sensing pillar arrays for biological measurements. Lab Chip 9, 1449-1454. Abstract Article

Duffy, D.C., McDonald, J.C., Schueller, O.J., and Whitesides, G.M. (1998). Rapid prototyping of microfluidic systems in poly(dimethylsiloxane). Anal. Chem. 70, 4974-4984. Abstract Article

Gilleland, C.L., Rohde, C.B., Zeng, F., and Yanik, M.F. (2010). Microfluidic immobilization of physiologically active Caenorhabditis elegans. Nat. Protoc. 5, 1888-1902. Abstract Article

Gray, J.M., Karow, D.S., Lu, H., Chang, A.J., Chang, J.S., Ellis, R.E., Marletta, M.A., and Bargmann, C.I. (2004). Oxygen sensation and social feeding mediated by a C. elegans guanylate cyclase homologue. Nature 430, 317-322. Abstract Article

Guo, S.X., Bourgeois, F., Chokshi, T., Durr, N.J., Hilliard, M.A., Chronis, N., and Ben-Yakar, A. (2008). Femtosecond laser nanoaxotomy lab-on-achip for in vivo nerve regeneration studies. Nat. Methods 5, 531-533. Abstract Article

Hulme, E.S., Shevkoplyas, S.S., and Whitesides, G.M. (2009). Incorporation of prefabricated screw, pneumatic, and solenoid valves into microfluidic devices. Lab Chip 9, 79-86. Abstract Article

Hulme, S.E., Shevkoplyas, S.S., Apfeld, J., Fontana, W., and Whitesides, G.M. (2007). A microfabricated array of clamps for immobilizing and imaging C. elegans. Lab Chip 7, 1515-1523. Abstract Article

Hulme, S.E., Shevkoplyas, S.S., Mcguigan, A.P., Apfeld, J., and Whitesides, G.M. (2010). Lifespan-on-a-chip: microfluidic chambers for performing lifelong observation of $C$. elegans. Lab Chip 10, 589-597. Abstract Article

Hulme, S.E., Shevkoplyas, S.S., and Samuel, A. (2008). Microfluidics: streamlining discovery in worm biology. Nat. Methods 5, 589-590. Abstract Article

Kim, N., Dempsey, C.M., Zoval, J.V., Sze, J.Y., and Madou, M.J. (2007). Automated microfluidic compact disc (CD) cultivation system of Caenorhabditis elegans. Sensors and Actuators B-Chemical 122, 511-518. Article

Krajniak, J., and Lu, H. (2010). Long-term high-resolution imaging and culture of C. elegans in chip-gel hybrid microfluidic device for developmental studies. Lab Chip 10, 1862-1868. Abstract Article

Lee, H., Crane, M.M., Zhang, Y., and Lu, H. (2013). Quantitative screening of genes regulating tryptophan hydroxylase transcription in Caenorhabditis elegans using microfluidics and an adaptive algorithm. Integr. Biol. (Camb). 5, 372-380. Abstract Article

Lockery, S. (2007). Channeling the worm: microfluidic devices for nematode neurobiology. Nat. Methods 4, 691-692. Abstract Article

Lockery, S.R., Hulme, S.E., Roberts, W.M., Robinson, K.J., Laromaine, A., Lindsay, T.H., Whitesides, G.M., and Weeks, J.C. (2012). A microfluidic device for whole-animal drug screening using electrophysiological measures in the nematode C. elegans. Lab Chip 12, 2211-2220. Abstract Article 
Lockery, S.R., Lawton, K.J., Doll, J.C., Faumont, S., Coulthard, S.M., Thiele, T.R., Chronis, N., McCormick, K.E., Goodman, M.B., and Pruitt, B.L. (2008). Artificial dirt: microfluidic substrates for nematode neurobiology and behavior. J. Neurophysiol. 99, 3136-3143. Abstract Article

Lucchetta, E.M., Lee, J.H., Fu, L.A., Patel, N.H., and Ismagilov, R.F. (2005). Dynamics of Drosophila embryonic patterning network perturbed in space and time using microfluidics. Nature 434, 1134-1138. Abstract Article

McCormick, K.E., Gaertner, B.E., Sottile, M., Phillips, P.C., and Lockery, S.R. (2011). Microfluidic devices for analysis of spatial orientation behaviors in semi-restrained Caenorhabditis elegans. PLoS One 6, e25710. Abstract Article

McDonald, J.C., Duffy, D.C., Anderson, J.R., Chiu, D.T., Wu, H.K., Schueller, O.J., and Whitesides, G.M. (2000). Fabrication of microfluidic systems in poly(dimethylsiloxane). Electrophoresis 21, 27-40. Abstract

McGrath, P.T., Rockman, M.V., Zimmer, M., Jang, H., Macosko, E.Z., Kruglyak, L., and Bargmann, C.I. (2009). Quantitative mapping of a digenic behavioral trait implicates globin variation in C. elegans sensory behaviors. Neuron 61, 692-699. Abstract Article

Park, S., Hwang, H., Nam, S.-W., Martinez, F., Austin, R.H., and Ryu, W.S. (2008). Enhanced Caenorhabditis elegans locomotion in a structured microfluidic environment. PLoS One 3, e2550. Abstract Article

Pincus, Z., Smith-Vikos, T., and Slack, F.J. (2011). MicroRNA predictors of longevity in Caenorhabditis elegans. PLoS Genet. 7, e1002306. Abstract Article

Qin, J., and Wheeler, A.R. (2007). Maze exploration and learning in C. elegans. Lab Chip 7, 186-192. Abstract Article

Rohde, C.B., Zeng, F., Gonzalez-Rubio, R., Angel, M., and Yanik, M.F. (2007). Microfluidic system for on-chip high-throughput whole-animal sorting and screening at subcellular resolution. Proc. Natl. Acad. Sci. U. S. A. 104, 13891-13895. Abstract Article

Samara, C., Rohde, C.B., Gilleland, C.L., Norton, S., Haggarty, S.J., and Yanik, M. (2010). Large-scale in vivo femtosecond laser neurosurgery screen reveals small-molecule enhancer of regeneration. Proc. Natl. Acad. Sci. U. S. A. 107, 18342-18347. Abstract Article

Shi, W., Qin, J., Ye, N., and Lin, B. (2008). Droplet-based microfluidic system for individual Caenorhabditis elegans assay. Lab Chip 8, 1432-1435. Abstract Article

Shi, W., Wen, H., Lu, Y., Shi, Y., Lin, B., and Qin, J. (2010). Droplet microfluidics for characterizing the neurotoxin-induced responses in individual Caenorhabditis elegans. Lab Chip 10, 2855-2863. Abstract Article

Sia, S., and Whitesides, G. (2003). Microfluidic devices fabricated in poly(dimethylsiloxane) for biological studies. Electrophoresis 24, 3563-3576. Abstract Article

Stirman, J.N., Brauner, M., Gottschalk, A., and Lu, H. (2010). High-throughput study of synaptic transmission at the neuromuscular junction enabled by optogenetics and microfluidics. J. Neurosci. Methods 191, 90-93. Abstract Article

Unger, M.A., Chou, H.P., Thorsen, T., Scherer, A., and Quake, S.R. (2000). Monolithic microfabricated valves and pumps by multilayer soft lithography. Science 288, 113-116. Abstract Article

Wen, Q., Po, M.D., Hulme, E., Chen, S., Liu, X., Kwok, Sen W., Gershow, M., Leifer, Andrew M., Butler, V., Fang-Yen, C., et al. (2012). Proprioceptive coupling within motor neurons drives C. elegans forward locomotion. Neuron 76, 750-761. Abstract Article

Whitesides, G.M. (2006). The origins and the future of microfluidics. Nature 442, 368-373. Abstract Article

Xia, Y.N., and Whitesides, G.M. (1998). Soft lithography. Ann. Rev. Mater. Sci. 28, 153-184. Article 
Yanik, M.F., Rohde, C.B., and Pardo-Martin, C. (2011). Technologies for micromanipulating, imaging, and phenotyping small invertebrates and vertebrates. Annu. Rev. of Biomed. Eng. 13, 185-217. Abstract Article

Zeng, F., Rohde, C.B., and Yanik, M.F. (2008). Sub-cellular precision on-chip small-animal immobilization, multi-photon imaging and femtosecond-laser manipulation. Lab Chip 8, 653-656. Abstract Article

Zhang, Y., Lu, H., and Bargmann, C.I. (2005). Pathogenic bacteria induce aversive olfactory learning in Caenorhabditis elegans. Nature 438, 179-184. Abstract Article

Zimmer, M., Gray, J.M., Pokala, N., Chang, A.J., Karow, D.S., Marletta, M.A., Hudson, M L., Morton, D.B., Chronis, N., and Bargmann, C.I. (2009). Neurons detect increases and decreases in oxygen levels using distinct guanylate cyclases. Neuron 61, 865-879. Abstract Article

All WormBook content, except where otherwise noted, is licensed under a Creative SOMRERIGHISRESERVED Commons Attribution License. 